\title{
Surgical antibiotic prophylaxis in a tertiary care teaching hospital in India
}

\author{
H. S. Rehan ${ }^{1}$, Ashish Kumar Kakkar', Shipra Goel ${ }^{2}$ \\ ${ }^{1}$ Department of Pharmacology, Lady Hardinge Medical College and SSK Hospital, New Delhi-110001 \\ ${ }^{2}$ Sixth Semester MBBS, Lady Hardinge Medical College and SSK Hospital, New Delhi-110001
}

doi: 10.3396/ijic.V6i2.014.10

\begin{abstract}
Appropriate antibiotic prophylaxis can reduce the risk of postoperative wound infections, but misuse and overuse of antimicrobials increases both the cost and the selection pressure favouring the emergence of resistant bacteria. Our main objective was to study the pattern of prophylactic antibiotics in different surgeries in a large tertiary care teaching hospital. A total of one hundred patients undergoing surgery, admitted under various hospital departments were enrolled in this prospective observational study. Pretested proforma which included information on patient characteristics, choice of antimicrobial agents as well as their route, timing and total duration of prophylaxis were completed. Also the appropriateness of antibiotic prophylaxis was assessed as per standard guidelines. It was found that third generation cephalosporins were the most commonly prescribed class of antibiotics. Although only $67 \%$ of cases required prophylaxis, it was utilised in $86 \%$ of procedures, being unjustified in 19\% of cases. Mean timing of administration of antibiotics was $3.22 \pm 1.03$ hours prior to first incision and the patients received post operative antibiotics for a mean duration of 5 days during their hospital stay. Thus, surgical prophylaxis was inappropriate in terms of choice of antimicrobial agent, timing of administration as well as the total duration of prescription, in majority of the cases. Interventions are warranted to promote the development, dissemination and adoption of evidence based guidelines for antimicrobial prophylaxis.
\end{abstract}

\section{Key Words}

antibiotic prophylaxis, surgery, surgical site infection

\section{Corresponding author}

Dr Ashish Kumar Kakkar, Department of Pharmacology, Lady Hardinge Medical College

New Delhi-110001. Email: drashishkr@rediffmail.com, Ph no.+ 919868051003 


\section{Introduction}

Surgical site infection (SSI) is the most common post operative complication and represents a significant burden in terms of patient morbidity and mortality, and cost to health services around the world. SSIs are also the second commonest nosocomial infection accounting for approximately one quarter of 2 million hospital acquired infections in USA annually. ${ }^{1,2}$ Among an estimated 27 million surgical procedures, surgical site infections are reported in up to 500,000 cases each year. ${ }^{3}$ It has been estimated that $2-5 \%$ of patients undergoing clean extra abdominal surgeries and up to $20 \%$ of patients undergoing intra abdominal procedures will develop a SSI. ${ }^{4-9}$

Appropriate antibiotic prophylaxis has been shown to be effective in reducing the incidence of surgical site infections. Selection of an appropriate antimicrobial agent (AMA) depends on the pathogen most likely to cause an infection. A single pre operative antibiotic dose is sufficient for operations lasting up to $4 .{ }^{10}$ In prolonged surgeries, however, further antibiotic doses may be needed to maintain the drug levels. Re-administration should also be considered in the event of prolonged or excessive intraoperative bleeding. Timing of antibiotic prophylaxis is considered optimal if administered within 30 minutes prior to incision. ${ }^{11}$ However, vancomycin or fluoroquinolone antibiotics should be given within 2 hours before the first surgical skin incision.

Some data suggest that nearly $30-50 \%$ of antibiotics used in hospitals are prescribed for surgical prophylaxis and $30-90 \%$ of this prophylaxis is inappropriate. ${ }^{12}$ Antimicrobial agents are overprescribed, given at a wrong time or continued for a long duration. ${ }^{12} \mathrm{~A}$ previous study in India had shown that approximately 55\% of the surgeons prescribed a single antibiotic for clean surgeries. A combination of two or three antimicrobial agents was preferred in clean contaminated $(42.3 \%)$ and dirty $(46.9 \%)$ surgeries respectively. Third generation cephalosporins (particularly ceftriaxone and cefotaxime) were the commonly prescribed antibiotics $(80 \%)$ for all surgeries. However, in majority of cases antibiotics were prescribed for durations longer than recommended in guidelines. ${ }^{13}$ Inappropriate use of antibiotics is associated with unnecessary increase in the cost of therapy and in the emergence of drug resistant bacteria. ${ }^{14}$
In India, due to lack of adequate information and guidelines for antimicrobial prophylaxis in surgery there is a need to generate baseline data on the pattern of use of prophylactic antibiotics. The aim of this study was to assess the current use of perioperative antimicrobials among patients undergoing surgical procedures in a tertiary care hospital.

\section{Materials and Methods}

A prospective observational study was conducted in Lady Hardinge Medical College and Smt. S. K. Hospital, New Delhi with the prior approval of the Institutional Review Board. All consecutive patients of any age undergoing surgery (elective as well as emergency) in the departments of general surgery, orthopedics, gynecology and obstetrics, ophthalmology and otorhinolaryngology were eligible for inclusion into the study. Patients with current infections prior to surgery were excluded.

Data were collected on a pretested case record form which included information on patient characteristics, surgical department under which admitted, type of operation, antimicrobial agents prescribed as well as their route of administration and timing of administration prior to surgery. Follow up data included additionally administered doses of antimicrobial agents, the total duration of prophylaxis as well as signs and symptoms of surgical site infections. If more than one drug was prescribed for a single procedure, all parameters for each drug were evaluated separately. The investigators did not intervene in patients' care in any way.

Appropriateness of preoperative antibiotic prophylaxis was assessed as per guidelines of Scottish Intercollegiate Guideline Network (SIGN) and American Society of Health-system Pharmacists (ASHP). ${ }^{11}{ }^{15}$ These guidelines provide evidence based recommendations to the practitioners for rational use of prophylactic antimicrobials.

\section{Results}

A total of 100 (51 male \& 49 female) patients were included in the study. Mean age of patients was 32.86 \pm 16.46 years (Range $6-78$ years). The great majority $(80 \%)$ of patients had an elective procedure while $20 \%$ were operated on an emergency basis. Most patients were admitted under General surgery department 


\section{Table I: Speciality/ Ward wise distribution of preoperative cases $(\mathrm{N}=100)$}

\begin{tabular}{ll} 
Speciality/ward & No. of cases \\
\hline General surgery & 30 \\
\hline Orthopedics & 26 \\
\hline Gynecology and obstetrics & 18 \\
\hline Otorhinolaryngology & 20 \\
\hline Ophthalmology & 6 \\
\hline
\end{tabular}

(30\%) followed by orthopaedics department (26\%) [Table I]. Inguinal hernia repair was the most frequent surgical procedure performed (14\%). All the patients undergoing hernia repair were prescribed preoperative antibiotics, most commonly co-amoxiclav. Similarly, all patients undergoing Caesarean section $(12 \%$ of total cases) received preoperative antibiotics. Coamoxiclav with metronidazole was the most commonly prescribed combination in this group followed by ampicillin alone. All patients with closed fractures (14 cases) received antibiotic prophylaxis, cefotaxime plus gentamicin was prescribed in majority of these cases.

As per SIGN and ASHP guidelines, prophylactic antimicrobials were indicated in $67 \%$ of all cases but were administered to $86 \%$ patients, being inappropriate in $19 \%$ cases especially clean surgeries such as herniorrhaphy, ocular surgeries and tonsillectomy.

A total number of 162 antibiotics were prescribed in our study. The most frequently prescribed classes of antibiotics were cephalosporins (42\%) followed by penicillins $(34 \%)$. Fifteen different combinations of antimicrobial drugs were used. Twenty eight percent of patients (24 cases) received a single drug for prophylaxis while 35 patients $(41 \%)$ received two drugs. Sixteen patients $(19 \%)$ received three drugs and one received 4 drugs (cefotaxime, metronidazole, ampicillin \& gentamicin) for prophylaxis. No preoperative prophylactic antibiotic was prescribed in 14 of 20 otorhinolaryngology cases.
Table II: Commonly prescribed single antimicrobial agents for prophylaxis $(\mathrm{N}=28)$

\begin{tabular}{ll} 
Antimicrobial agent & No. of cases \% \\
\hline Co-amoxiclav & $12(42.8)$ \\
\hline Ciprofloxacin & $8(28.6)$ \\
\hline Ampicillin & $4(14.3)$ \\
\hline Ceftriaxone & $3(10.7)$ \\
\hline Cefotaxime & $1(3.6)$
\end{tabular}

Co-amoxiclav, a fixed dose combination of amoxicillin \& clavulanic acid was the most commonly prescribed single agent for prophylaxis $(12 \%)$ followed by ciprofloxacin (8\%) and ampicillin (4\%) [Table II].

Among the antibiotic combinations, commonly used regimens included cefotaxime and gentamicin (12 patients). Cefatoxime, gentamicin \& metronidazole (12 patients) followed by co-amoxiclav \& metronidazole (8 patients) [Table III].

Mean time of administration of prophylactic antibiotics was $3.22 \pm 1.03$ hours prior to surgery. Longest mean duration between drug administration \& surgical skin incision was recorded for ampicillin ( $5 \mathrm{hrs})$ \& shortest for gentamicin (1.5 hrs) [Table IV] $13 \%$ patients received antibiotics just before incision. All preoperative antibiotics were administered by intravenous route except in ocular surgeries where oral and topical antimicrobials were prescribed.

The study patients received post operative antibiotics for a mean duration of 5 days during their stay in hospital plus 6 days following discharge from ward/unit. Few patients $(12 \%)$ complained of purulent discharge at incision site and surgical site infection was reported in 14 percent of cases. The use of antimicrobials in all the cases was empirical based on operating surgeon's clinical experience. One of the study patients who was prescribed a combination of ampicillin, amikacin and metronidazole experienced nausea. No other adverse drug reaction was reported in the study. 
Table III: Frequently used combinations of antimicrobial agents for surgical prophylaxis $(\mathrm{N}=58)$

\section{Combinations}

1. Two drug combinations

Co-amoxiclav + metronidazole

Cefotaxime + gentamicin

Ceftriaxone + metronidazole

Cefotaxime + metronidazole

Ciprofloxacin + metronidazole

Ampicillin + metronidazole

Co-amoxiclav + gentamicin

Ceftriaxone + gentamicin

Amoxicillin + gentamicin

2. Three drug combinations

Cefotaxime + metronidazole + gentamicin

Ciprofloxacin + metronidazole + co-amoxiclav

Penicillin + metronidazole + amikacin

Ampicillin + amikacin + metronidazole

Cefotaxime + metronidazole + gentamicin

3. Four drug combination

Cefotaxime + ampicillin + gentamicin + metronidazole

\section{No. of cases}

$$
N=41
$$

\section{Discussion}

The effectiveness of preoperative antibiotic prophylaxis is well established. Despite this, surveys have shown that optimal practice isn't achieved in many hospitals. ${ }^{16}$ The majority (86\%) of patients received antibiotic prophylaxis prior to surgery. This figure is comparable to those reported in previous studies from Turkey, Israel and Greece. ${ }^{17-19}$ Among the study participants 58\% received antimicrobial combinations. Combinations of antibiotics were used in all the surgical departments and $17 \%$ patients received three or more drugs together. Although the use of two or more antimicrobials in combination may have a certain rationale, their indiscriminate use can have several negative consequences. Potentially harmful aspects of such inappropriate antibiotic combinations include the emergence of resistant bacteria, super-infection, the risks of toxic and allergic reactions and increased cost of therapy.

Selection of antibiotic was inappropriate in all patients operated for caesarean delivery and gall stone disease. Noneof the patients received cefazolin as recommended by ASHP guidelines. Further all patients undergoing hernia repair received preoperative antibiotics while no such prophylaxis is recommended as per SIGN guidelines. Fourteen patients admitted under the Otorhinolaryngology department, who underwent a 'clean' surgery, did not receive any antibiotic 
Table IV: Timing of administration of prophylactic antimicrobials

Antimicrobial Agent

Mean timing of administration

prior to surgery (hours)

\begin{tabular}{lc}
\hline Ceftriaxone & 3 \\
\hline Co-amoxiclav & 3.5 \\
\hline Ciprofloxacin & 2.5 \\
\hline Ampicillin & 5 \\
\hline Penicillin & 4 \\
\hline Cefotaxime & 2.5 \\
\hline Metronidazole & 3 \\
\hline Amikacin & 4 \\
\hline Gentamicin & 1.5 \\
\hline
\end{tabular}

prophylaxis as recommended. Patients undergoing ophthalmic procedures received additional oral antibiotics while topical route is the primary mode of prophylaxis recommended in such patients. ${ }^{15}$ Routine prophylactic use of systemic antibiotics is generally not recommended for cataract surgery. ${ }^{20}$

In our study, third generation cephalosporins were prescribed to almost half of all the patients who received preoperative antibiotics (42 of 86), which was inappropriate as per SIGN and ASHP guidelines. Thirty two percent of study participants received cefotaxime and $10 \%$ received ceftriaxone prior to their surgery. For surgical prophylaxis it is important to select an antibiotic with narrowest antibacterial spectrum to reduce the emergence of resistance and also because broad spectrum antibiotics may be required later if patient develops serious sepsis. Therefore, it is recommended that the use of third generation cephalosporins such as ceftriaxone and cefotaxime be avoided in surgical prophylaxis. ${ }^{21}$

According to SIGN guidelines, all antibiotic administration must be completed at time of surgical incision and no more than 30 minutes prior. In our study antibiotics were administered at least $1 \frac{1}{1} 2$ hours prior to surgery. Only 13 percent of patients received antibiotics just before incision.

As far as duration of antimicrobial prophylaxis is concerned, one antibiotic dose is sufficient for operations lasting 4 hours or less. ${ }^{10}$ Further doses may be required in prolonged surgeries. Results from two large studies highlight the fact that single dose antibiotic prophylaxis is not associated with an increased rate of SSI when compared to multiple dose regimens. ${ }^{22,23}$ Persistence of tissue concentrations past the period of surgery and recovery from anaesthesia doesn't improve efficacy and increases toxicity and cost of therapy. ${ }^{21}$ In our study, post operative antibiotics were administered for a mean duration of 5 days during hospital stay and another 6 days following discharge, a period much longer than recommended. A mean duration of 6.4 days of antibiotic use has been reported in a study of Taiwanese patients. ${ }^{24}$ Even though evidence from literature fails to support prolonged administration of AMAs in the hospitals, usage beyond 24 hours is common. ${ }^{25}$ Longer courses of antibiotics are falsely believed to be a good preventive measure against SSIs. Various studies assessing the appropriateness of surgical antibiotic prophylaxis have shown variable results - 95\% (Israel), ${ }^{26} 75 \%$ (Brazil) ${ }_{1}^{27} 5.9 \%\left(\right.$ Iran ${ }^{28}$ 


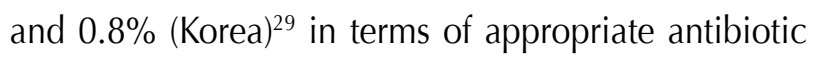
choice. Since preoperative prophylactic antibiotics take up a large part of prescribed AMAs in the hospitals, adherence to standard guidelines regarding the duration of antibiotic prophylaxis would keep costs to a minimum which would be desirable especially in a resource limited setting like ours.

The results highlight the challenges of disseminating evidence based protocols systematically into routine clinical practice. Various measures are needed to improveappropriateness of prescriptions and adherence include development of evidence based guidelines in collaboration with surgeons, increased outcomebased research to document benefits of appropriate antibiotic use, continuing education to disseminate information to practitioners, surveys of antibiotic use and reassessment of prescribing practices over time and providing regular feedback and organizing group education and consensus meetings.

\section{References}

1. MartoneWJ, Nochols RL. Recognition, prevention, surveillance and management of surgical site infections. Introduction to the problem and symposium overview. Clin Infect Dis 2001; Suppl 33: 67-68.

2. Haley RW, Culver DH, White JW, Morgan WM, Emori TG. The nationwide nosocomial infection rate: a new need for vital statistics. Am J Epidemiol 1985; 121: 159-167.

3. Centers for Disease Control and Prevention, National Center for Health Statistics Vital and Health Statistics, Detailed diagnoses and procedures national hospital discharge survey 1994. Vol 127. Hyattsville (MD): Department of Health and Human Services; 1997.

4. Delgado-Rodriguez $M$, Sillero-Arenas $M$, Medina-Cuadros $M$, Martinez-Gallego G. Nosocomial infections in surgical patients: comparison of two measures of intrinsic patient risk. Infect Control Hosp Epidemiol 1997; 18: 19-23.

5. Horan TC, Culver DH, Gaynes RP, Jarvis WR, Edwards JR, Reid CR. Nosocomial infections in surgical patients in the United States, January 1986-June 1992. National Nosocomial Infections Surveillance (NNIS) System. Infect Control Hosp Epidemiol 1993; 14: 73-80.

6. Horan TC, Gaynes RP, Martone WJ, Jarvis WR, Emori TG. CDC definitions of nosocomial surgical site infections, 1992: a modification of CDC definitions of surgical wound infections. Infect Control Hosp Epidemiol 1992; 13: 606-608.

7. Horan TC, Emori TG. Definitions of key terms used in the NNIS System. Am I Infect Control 1997; 25: 112-116.

8. Wallace WC, Cinat M, Gornick WB, Lekawa ME, Wilson SE. Nosocomial infections in the surgical intensive care unit: a difference between trauma and surgical patients. Am Surg 1999; 65: 987-990.

9. Scheel O, Stormark M. National prevalence survey on hospital infections in Norway. J Hosp Infect 1999; 41: 331-335.

10. McDonald M, Grabsch E, Marshall C, Forbes A. Single-versus multiple-dose antimicrobial prophylaxis for major surgery: a systematic review. Aust N Z J Surg 1998; 68: 388-396.
11. Scottish Intercollegiate Guidelines Network. Antibiotic prophylaxis in surgery. Available from URL: http:// www.sign. ac.uk/pdf/sign104.pdf [cited 2009 Mar 5].

12. Dettenkofer M, Forster DH, Ebner W, Gastmeier P, Ruden $\mathrm{H}$, Daschner FD. The practice of perioperative antibiotic prophylaxis in eight German hospitals. Infection 2002; 30: 164-167.

13. Kulkarni RA, Kochhar PH, Dargude VA, Rajadhyakshya SS, Thatte UM. Patterns of antimicrobial use by surgeons in India. Ind I Surg 2005; 67: 308-315.

14. Nichols RL. Preventing surgical site infections: A surgeon's perspective. Emerg Infect Dis 2001; 7(2): 220-224.

15. American Society of Health System Pharmacists. The ASHP therapeutic guidelines on antimicrobial prophylaxis in surgery. Available from URL:http://www.ashp.org/DocLibrary/ BestPractices/TG_Surgical.aspx [cited 2009 Mar 11].

16. Dellinger EP, Hausmann S, Bratzler D et al. Hospitals collaborate to decrease surgical site infections. Am / Surg 2005; 190: 9-15.

17. Yalcin AN, Serin S, Gurses E, Zencir M. Surgical Antibiotic Prophylaxis in Turkish University Hospital. J Chemother 2002; 14: 373-377.

18. Finkelstein R, Reinhertz G, Embom A. Surveillance of use of antibiotic prophylaxis in surgery. Isr J Med Science 1996; 32: 1093-1097.

19. Tourmousoglou CE, Yiannakopoulou ECh, Kalapothaki V, Bramis J, St Papadopoulos J. Adherence to guidelines for antibiotic prophylaxis in general surgery: a critical appraisal. J Antimicrob Chemother. 2008; 61(1): 214-218.

20. Starr MB. Prophylactic antibiotics for ophthalmic surgery. Surv Ophthalmol 1983; 27(6): 353-373.

21. Munckhof W. Antibiotics for surgical prophylaxis. Aust Prescr 2005; 28: 38-40.

22. Kanayama M, Hashimoto T, Shigenobu K, Oha F, Togawa D. Effective prevention of surgical site infection using a Centers for Disease Control and Prevention guideline-based antimicrobial prophylaxis in lumbar spine surgery. I Neurosurg Spine. 2007; 6(4): 327-329.

23. Fonseca SN, Kunzle SR, Junqueira MJ, Nascimento RT, de Andrade JI, Levin AS. Implementing 1-dose antibiotic prophylaxis for prevention of surgical site infection. Arch Surg 2006; 141(11): 1109-1113.

24. Chen YS, Liv YH, Kunin CM, Huang JK, Tsai CC. Use of prophylactic antibiotics in surgery at a Medical centre in southern Taiwan. J Formos Med Assoc 2002; 101: 741-748.

25. Bratzler DW, Houck PM, Richards C et al. Use of antimicrobial prophylaxis for major surgery: baseline results from the National Surgical Infection Prevention Project. Arch Surg 2005; 140: 174-182.

26. Vaisbrud V, Raveh D, Schlesinger Y, Yinnon AM. Surveillance of antimicrobial prophylaxis for surgical procedures. Infect Control Hosp Epidemiol 1999; 20(9): 610-613.

27. Heineck I, Ferreira MBC, Schenkel EP. Prescribing practice for antibiotic prophylaxis for 3 commonly performed surgeries in a teaching hospital in Brazil. Am J Infect Control 1999; 27: 296-300.

28. Askarian M, Moravveji AR, Mirkhani H, Namazi S, Weed H. Adherence to American Society of Health-System Pharmacists surgical antibiotic prophylaxis guidelines in Iran. Infect Control Hosp Epidemiol. 2006; 27(8): 876-878.

29. Choi WS, Song JY, Hwang JH, Kim NS, Cheong HJ. Appropriateness of antibiotic prophylaxis for major surgery in Korea. Infect Control Hosp Epidemiol. 2007; 28(8): 9971002. 